\title{
Numerical Study of Hydrogen Trapping: Application to an API 5L X60 Steel
}

\author{
Patricia Castaño-Rivera, ${ }^{1}$ Viviana P. Ramunni, ${ }^{1}$ and Pablo Bruzzoni ${ }^{2}$ \\ ${ }^{1}$ Materials Division, Hydrogen in Metals Group, Instituto de Tecnología Jorge Sabato, Av. General Paz 1499, B1650KNA, \\ San Martín, Prov. de Buenos Aires, C1033AAJ Buenos Aires, Argentina \\ ${ }^{2}$ CNEA/CAC, UAM, Avenida General Paz 1499, CP1650 San Martín, Argentina \\ Correspondence should be addressed to Patricia Castaño-Rivera, pcastanio@cnea.gov.ar
}

Received 4 May 2012; Accepted 12 June 2012

Academic Editors: J. Foct, K. Kusabiraki, and M. Nazmy

Copyright (๑) 2012 Patricia Castaño-Rivera et al. This is an open access article distributed under the Creative Commons Attribution License, which permits unrestricted use, distribution, and reproduction in any medium, provided the original work is properly cited.

A numerical finite difference method is developed here to solve the diffusion equation for hydrogen in presence of trapping sites. A feature of our software is that an optimization of diffusion and trapping parameters is achieved via a non linear least squares fit. On the other hand, we have demonstrated that usual electrochemical hydrogen permeation tests are enough to assess hydrogen free energies of trapping in the range of $-35 \mathrm{~kJ} / \mathrm{mol}$ to $-70 \mathrm{~kJ} / \mathrm{mol}$. These conclusions are obtained by assuming the presence of saturable traps in local equilibrium with hydrogen and are validated by means of simulated permeation and degassing transients. In addition, we check our model performing electrochemical hydrogen permeation tests at $30^{\circ} \mathrm{C}, 50^{\circ} \mathrm{C}$, and $70^{\circ} \mathrm{C}$, on an $\mathrm{API} 5 \mathrm{~L}$ $\mathrm{X} 60$ as received steel state to study its trapping and diffusion properties considering only one type of trapping site. The binding energies $(\Delta G)$ and the trap densities $(N)$ are determined by fitting the theoretical model to the experimental permeation data. The steel presents a high density of weak traps, $|\Delta G|<35 \mathrm{KJ} / \mathrm{mol}$, namely, $N=1.4 \times 10^{-5} \mathrm{~mol} \mathrm{~cm}^{-3}$. Strong trapping sites which alter the shape of the permeation transient are also detected; their $\Delta G$ values ranged from 57 to $72 \mathrm{KJ} / \mathrm{mol}$.

\section{Introduction}

In the literature it is possible to find different approaches to fit simulated hydrogen permeation transients to experimental ones, generally based on the model of McNabb and Foster [1]. Nowadays, this formulation is the most accepted model of hydrogen permeation in presence of one type of traps, with kinetic trapping and detrapping parameters $k$ and $p$, respectively. The traps are saturable, that is, each trap is a site which allows only one hydrogen atom. Thus, only two states are conceivable for such trap: empty or occupied. The interaction between different trapped hydrogen atoms is assumed negligible. Reference [1] supplies analytical expressions for the time lag as a function of $k, p$ and the diffusion coefficient $D$. However, no analytical expressions for the permeation transient (hydrogen flux at the exit side of the permeation membrane as a function of time) are given, since the presented coupled differential equations are not solved. The $\mathrm{McNabb}$ and Foster equations are solved numerically (finite difference method) by Caskey and Pillinger [2] and later by Thomas and Stern [3]. Simulated permeation transients are obtained for different sets of parameters $D, k, p$, and $N_{x}$, where $N_{x}$ is the trap population measured either in sites per unit volume or in moles per unit volume. These authors [2, 3] do not compare simulated transients with experimental ones. One attempt to fit simulated permeation transients to experimental data is that of Johnson and Lin [4], which assumes the simplification of local equilibrium theory proposed in 1970 by Oriani [5]. The experimental data correspond to hydrogen permeation through cold rolled iron with gas phase charging and electrochemical detection, and the discussion is centered on whether the traps are saturable or not. A subsequent remarkable work on fitting of simulated transients to experimental data is performed by Ferris and Turnbull [6]. They have studied the hydrogen permeation through a 430 martensitic stainless steel. An additional feature to the McNabb and Foster formulation is introduced, that is, the presence of irreversible traps is also considered. 
They have calculated the kinetic parameters of trapping and detrapping. However, the value of these parameters has not been validated yet through independent measurements. Following the formulation and methods of Ferris and Turnbull, Ramunni et al. obtained trapping parameters for a steel with different microstructures, where the cementite morphology is changed through selected thermal treatments [7]. Although they are able to simulate permeation transients covering the whole possibilities of the McNabb and Foster formulations, they restricted themselves to the case of local equilibrium, by setting the kinetic parameters high enough to guarantee a fast hydrogen exchange between traps and normal lattice sites. Concerning to the nature of the trapping sites in a steel and their free energy of trapping, it has been repeatedly pointed out that different values of trapping energy are expected. Dislocations can trap hydrogen with different binding energies depending on whether hydrogen is located at the dislocation core or in the stress field around a dislocation, whether edge or screw dislocations are involved, and so forth. Coherent precipitates like $\mathrm{TiC}$ can trap hydrogen in the distorted ferrite lattice around the precipitate with different trap energies [8]. In fact, it has been suggested that a full distribution of hydrogen trapping energies may exist, and this seems to be supported by the results of thermal desorption spectrometry [7], which is a very useful technique to assess hydrogen trapping energies. Briefly, through a numerical finite difference method, we want to solve the McNabb and Foster [1] differential equations in the case of unidirectional hydrogen flux:

$$
\begin{gathered}
\frac{\partial}{\partial t}\left(c+\sum_{i=1}^{Q} N_{i} \theta_{i}\right)=D_{L} \frac{\partial^{2} c}{\partial x^{2}}, \\
\frac{\partial \theta_{i}}{\partial t}=k c-p \theta_{i} ; \quad 1 \leq i \leq Q,
\end{gathered}
$$

where $x$ is the spatial coordinate along the diffusion direction, $c\left[\mathrm{~mol} / \mathrm{cm}^{3}\right]$ is the lattice hydrogen concentration, $N i$ the density of high energy $i$ type sites $\left[\mathrm{mol} / \mathrm{cm}^{3}\right]$ ( $Q$ different types are assumed), $i$ the fraction of occupied $i$ traps, $D_{L}\left[\mathrm{~cm}^{2} / \mathrm{s}\right]$ the lattice diffusion coefficient, $k$ $\left[\mathrm{s}^{-1} \mathrm{~cm}^{3} \mathrm{~mol}^{-1}\right]$ and $p\left[\mathrm{~s}^{-1}\right]$ are, respectively, the trapping and detrapping kinetic constants. Section 2 succinctly discusses the assumptions used to solve the diffusion equations, in particular the assumption of local equilibrium. In Section 3 we show that a single permeation transient is enough to describe trapping sites. Sections 4 and 5 describe the numerical method developed here to solve (1) and (2) and present a theoretical discussion in which we establish the range of trap energies which can be determined trough a single permeation transient. Then, we present simulated hydrogen permeation transients to support this conclusion. Section 6 presents the fitting of the present model with just one type of trap $(Q=1)$ to experimental permeation and degassing tests on an API 5L X60 steel as an example of application. We calculate the density of trapping sites and the hydrogen binding energy of $\mathrm{H}$ with traps. In Section 7 we check our software with another more general to solve the McNabb and Foster equations [7]. Finally, a brief conclusion to summarize the relevant points of the present work is presented.

\section{Theoretical Model and Assumptions}

The existence of saturable traps and the hypothesis of local equilibrium are assumed in the present work. According to the latter, the exchange of hydrogen between lattice sites and neighbor trap sites in iron or iron alloys is fast enough to achieve dynamic equilibrium for the trapping (3) and detrapping (4) reactions:

$$
\begin{gathered}
\mathrm{H}-L+X_{i} \longrightarrow \mathrm{H}-X_{i}+L ; \quad r_{T, i}=k_{i} c\left(1-\theta_{i}\right), \\
\mathrm{H}+L-X_{i} \longrightarrow \mathrm{H}+X_{i}-L ; \quad r_{D, i}=p_{i} \theta_{i} N_{L}\left(1-\theta_{L}\right),
\end{gathered}
$$

$L$ is a normal lattice site (tetrahedral interstice in bcc iron) with site density $N_{L}=0.85 \mathrm{~mol} \mathrm{~cm}^{-3}, X_{i}$ is a trap site of type $i, r_{T, i}$, and $r_{D, i}$ are the individual trapping and detrapping rates of hydrogen in a type $i$ trap in $\left[\mathrm{s}^{-1}\right], k_{i}\left[\mathrm{~s}^{-1} \mathrm{~cm}^{3} \mathrm{~mol}^{-1}\right]$ and $p_{i}\left[\mathrm{~s}^{-1}\right]$ are, respectively, the trapping and detrapping kinetic constants, $c\left[\mathrm{~mol} / \mathrm{cm}^{3}\right]$ is the lattice concentration of hydrogen, $\theta_{i}$ is the fraction of type $i$ traps which are bonded to hydrogen (or trap occupancy), $\left(1-\theta_{i}\right)$ is the fraction of empty traps of type $i, \theta_{L}$ is the fraction of normal lattice sites which are occupied by hydrogen and $\left(1-\theta_{L}\right)$ is the fraction of empty normal lattice sites. If dynamic equilibrium is achieved, the trapping and detrapping rates become equal: $r_{T, i}=r_{D, i}$. Then, using (3), (4), $K_{i}=k_{i} / p_{i}$ and assuming that $\theta_{L} \ll 1$, it results:

$$
K_{i}=\frac{\theta_{i}}{c\left(1-\theta_{i}\right)}
$$

here $K_{i}$ is the equilibrium constant for the trapping reaction, related to the standard free energy of trapping $\Delta G_{i}$ by the equation:

$$
\Delta G_{i}=-R T \ln K_{i}
$$

$T$ is the absolute temperature and $R$ is the universal constant gases. The reference states implicitly assumed in this definition are: for hydrogen in the lattice, dilute solution with $c=1 \mathrm{~mol} / \mathrm{cm}^{3}$; for trapped hydrogen, traps with $\theta_{i}=$ 0.5 . Under the assumption of local equilibrium, the trap occupancy can be expressed using (5) as a function of the lattice concentration $c$. Then the coupled $Q+1$ differential equations (1) and (2) can be reduced to a single differential one:

$$
\frac{\partial}{\partial t}\left(c+\sum_{i=1}^{R} N_{i} \frac{K_{i} c}{1+K_{i} c}\right)=D_{L} \frac{\partial^{2} c}{\partial x^{2}} .
$$

Equation (7) shows that, if local equilibrium is achieved, the local fraction of filled traps or trap occupancy $\theta_{i}$ depends on both, the lattice hydrogen concentration $c$ and the free energy of trapping $\Delta G_{i}$. The former is limited by the charging conditions at the entry side of the permeation membrane. For example, for gas phase charging in which equilibrium is 
achieved between gaseous hydrogen and absorbed hydrogen at the entry surface, Sievert's law holds:

$$
c_{0}=S \sqrt{p_{\mathrm{H}_{2}}},
$$

where $c_{0}$ is the lattice concentration of hydrogen just beneath the input surface (i.e., the maximum concentration of hydrogen throughout the permeation membrane), $S$ is the Sievert constant, and $p_{\mathrm{H}_{2}}$ is the partial pressure of hydrogen in the charging chamber. The Sievert constant is usually considered to depend on temperature according to:

$$
S=S_{0} \exp \left(\frac{-E_{S}}{R T}\right)
$$

here $S_{0}$ and $E_{S}$ are constants. Then, if the charging conditions are fixed, the value of the trap occupancy at the input surface $\theta_{i, 0}$ (i.e., the maximum value of $\theta_{i}$ throughout the membrane) depends only on $\Delta G_{i}$.

\section{Characterization of Traps through a Single Permeation Transient}

We discuss the range of $\Delta G_{i}$ values that can be assessed through a single hydrogen permeation experiment. We assume that only one type of trap is present $(Q=1)$ and for simplicity the index $i$ (trap type) is not specified. Then the trap occupancy is $\theta$, the trap density $N$, the free energy of trapping $\Delta G$ and the trapping equilibrium constant is $K=\exp (-\Delta G / R T)$. It is also assumed that hydrogen is introduced via gas phase charging with $p_{\mathrm{H} 2}=1$ bar and local equilibrium is achieved. If $\theta$ is negligible against unity, (5) shows that $\theta$ is proportional to $c$. The balance of diffusing hydrogen in a volume element leads to a differential equation similar to the second Fick's law, where the lattice diffusion coefficient $D_{L}$ is replaced by an apparent diffusion coefficient $D_{\text {app }}$. As pointed out by Oriani and assuming local equilibrium and $\theta \ll 1[5]$ :

$$
D_{\text {app }}=\frac{D_{L}}{1+K N},
$$

being $N$ the trap density $\left[\mathrm{mol} / \mathrm{cm}^{3}\right]$. Equation (7) can be solved analytically to yield the well known solution of hydrogen flux $J_{\mathrm{H}}$ as a function of time:

$$
J_{\mathrm{H}}=J_{\mathrm{H}, \mathrm{ss}}\left[1+2 \sum_{n=1}^{\infty} \exp \left(-n^{2} \pi^{2} \frac{D t}{L^{2}}\right)\right],
$$

here $J_{\mathrm{H}, \mathrm{ss}}=c_{0} D_{L} / L$ is the hydrogen flux at the steady state, $c_{0}$ is the lattice hydrogen concentration close to the input surface, $L$ is the thickness of the permeation membrane and $D$ is a diffusion coefficient. For the material without traps, $D$ is set as the lattice diffusion coefficient $D_{L}$. Assuming a density of $N$ traps in the material with trapping equilibrium constant $K, D$ is set as $D_{\text {app }}$, according to (10) as long as $\theta \ll 1$. Equation (10) shows that different combinations of $K$ and $N$ yield to the same value of $D_{\text {app}}$, and the expected permeation transient is the same for each of such combinations. Then, if the trap occupancy is negligible against unity a permeation transient at a single temperature is not able to assess the free energy trapping value. Let $\theta_{\min }$ be the upper limit of the trap occupancy value negligible against unity, from (10) the critical value of the equilibrium constant $K_{\min }$ is given by:

$$
K_{\min }=\frac{\theta_{\min }}{c\left(1-\theta_{\min }\right)} .
$$

To ensure that the condition $\theta \ll 1$ is achieved throughout the membrane, the critical location is the input surface. The lattice concentration at the input surface $c_{0}$ is fixed by the charging conditions as mentioned above. Then

$$
\begin{gathered}
K_{\min }=\frac{\theta_{\min }}{c_{0}\left(1-\theta_{\min }\right)}, \\
\left|\Delta G_{\min }\right|=R T \ln K_{\min } .
\end{gathered}
$$

With the above equations the minimum free energy of trapping can be determined in a permeation experiment at temperature $T$. The value of $c_{0}$ needed for the calculation of $\Delta G_{\min }$ depends on the hydrogen charging conditions. For gas phase charging, this value is obtained through (8) and (9). The constants $E_{S}$ and $S_{0}$ in (9) are obtained by combining hydrogen diffusion and permeation data from the literature $[9,10]: S_{0}=5.02 \times 10^{4} \mathrm{~mol} \mathrm{H} \mathrm{cm}^{-3} \mathrm{bar}^{-1 / 2}$ and $E_{S}=30.17 \mathrm{~kJ} / \mathrm{mol}$. The Sievert's constant $S$ is introduced in (8) together with $p_{\mathrm{H}_{2}}$ to obtain $c_{0}$. If the absolute value of the free energy trapping is high (deep traps), the traps tend to saturate with hydrogen and $\theta \sim 1$. In this case, a diffusion front develops. This diffusion front moves forward only when practically all traps behind it are filled. Then, when the absolute value of $\Delta G$ is higher than a certain critical value $\Delta G_{\max }$, the permeation transient for a given thickness, the lattice diffusion, and charging conditions will depend only on the density of traps and not on the free energy of trapping. The value of $\Delta G_{\max }$ can be calculated in a similar way as $\Delta G_{\min }$, by setting in this case a critical value of $\theta\left(\theta_{\max }\right)$ close to unity. Then $\theta_{\max }$ is the minimum value of $\theta$ which allows the determination of $\Delta G$ via a single permeation transient at a given temperature. Using a similar procedure but replacing $\theta_{\min }$ by $\theta_{\max }$ the value of $K_{\max }$ and $\Delta G_{\max }$ is calculated.

The critical absolute values of the free energy of trapping which can be assessed through a single permeation experiment $\Delta G_{\min }$ and $\Delta G_{\max }$ are calculated with equations (8), (9), (12), and (14) as a function of temperature. The chosen critical values of $\theta_{\min }$ and $\theta_{\max }$ are 0.002 and 0.998 , respectively. The hydrogen partial pressure is set as $p_{\mathrm{H}_{2}}=1$ bar. The results of these calculations are shown in Table 1. For the usual available temperatures in a permeation experiment with electrochemical detection, $\left|\Delta G_{\min }\right|$ is from ca. 33 to ca. $34 \mathrm{~kJ} / \mathrm{mol}$ and changes slightly with temperature. On the other hand $\left|\Delta G_{\max }\right|$ increases moderately with temperature from $63 \mathrm{~kJ} / \mathrm{mol}$ to $72 \mathrm{~kJ} / \mathrm{mol}$ showing that higher temperatures are best suited to determine high values of $\Delta G$. These limiting values $\Delta G_{\min }$ and $\Delta G_{\max }$ will change according to the charging conditions. Charging conditions which lead to higher values of $c_{0}$ (i.e., electrochemical charging, use of recombination poisons for hydrogen) cause a decrease of both $\Delta G_{\min }$ and $\Delta G_{\max }$. 
TABLE 1: Minimal and maximal absolute values of the free energy of trapping $\Delta G$.

\begin{tabular}{lcccc}
\hline $\begin{array}{l}\text { Temperature } \\
{ }^{\circ} \mathrm{C}\end{array}$ & $\begin{array}{c}C_{0} \\
10^{-9} \mathrm{~mol} \mathrm{H} \mathrm{cm}^{3}\end{array}$ & $\begin{array}{c}K_{\min } \\
(\mathrm{mol} \mathrm{H})^{-1} \mathrm{~cm}^{3}\end{array}$ & $\begin{array}{c}\left|\Delta G_{\min }\right| \\
\mathrm{KJ} / \mathrm{mol}\end{array}$ & $\begin{array}{c}K_{\max } \\
(\mathrm{mol} \mathrm{H})^{-1} \mathrm{~cm}^{3}\end{array}$ \\
\hline 10 & 1.36 & $1.47 \times 10^{-6}$ & 33.4 & $3.66 \times 10^{-11}$ \\
30 & 3.18 & $6.30 \times 10^{-5}$ & 33.7 & $1.57 \times 10^{-11}$ \\
50 & 6.67 & $3.01 \times 10^{-5}$ & 33.9 & $7.48 \times 10^{-10}$ \\
70 & 12.83 & $1.56 \times 10^{-5}$ & 34.1 & $3.89 \times 10^{-10}$ \\
90 & 22.97 & $8.73 \times 10^{-4}$ & 34.4 & 65.0 \\
\hline
\end{tabular}

\section{Theoretical Model}

The present computer simulation of the hydrogen permeation transients is based on the model of McNabb and Foster [1] of saturable hydrogen traps with simple kinetic laws for trapping and detrapping. Here, McNabb and Foster notation is partly changed. Assuming only one type of trap and unidirectional diffusion, the differential equation to solve is

$$
\frac{\partial C_{L}}{\partial t}+k_{T} c_{L}(1-\theta) N-p \theta N=D_{L} \frac{\partial^{2} c_{L}}{\partial x^{2}},
$$

where $c_{L}$ is the lattice $\mathrm{H}$ concentration, that is, the concentration of $\mathrm{H}$ in normal interstitial lattice sites (NILS), $t$ is time, $D_{L}$ is the lattice diffusion coefficient, $x$ is the spatial coordinate, $N$ is the trap density, $\theta$ is the fraction of occupied traps, and $k_{T}$ and $\rho$ are the trapping and detrapping kinetic constants, respectively. Equation (15) can be rewritten in terms of dimensionless variables:

$$
X=\frac{x}{L} ; \quad u=\frac{c_{L}}{C_{L 0}} ; \quad \omega=\frac{N \theta}{c_{L 0}},
$$

where $L$ and $c_{L 0}$ are, respectively, a characteristic dimension and a characteristic lattice $H$ concentration of the system. For the present case of $\mathrm{H}$ permeation through a metallic membrane, $L$ is the membrane thickness and $c_{L 0}$ is the lattice $\mathrm{H}$ concentration beneath the entry surface of the permeation membrane:

$$
\frac{\partial u}{\partial \tau}+\frac{N L^{2}}{D_{L}} k_{T} u-\frac{L^{2} p}{D_{L}} \omega-\frac{c_{L 0} L^{2} k_{T}}{D_{L}} u \omega=\frac{\partial^{2} u}{\partial X^{2}},
$$

or by defining the constants $\lambda, \mu$, and $\nu$ :

$$
\begin{gathered}
\frac{\partial u}{\partial \tau}+\lambda u-\mu \omega-\nu u \omega=\frac{\partial^{2} u}{\partial X^{2}}, \quad \lambda=\frac{N L^{2} k_{T}}{D_{L}}, \\
\mu=\frac{L^{2} p}{D_{L}}, \quad \nu=\frac{c_{L 0} L^{2} k_{T}}{D_{L}} .
\end{gathered}
$$

The hypothesis of local equilibrium proposed by Oriani [5] is assumed here. This hypothesis implies that the detrapping time constant $(1 / \rho)$ is much lower than the diffusion time constant $\left(L^{2} / D_{L}\right)$. As reported in the next paragraphs, in the present work it has been empirically found that the choice of $1 / \lambda \leq 0.001 L^{2} D_{L}$ (i.e., $\mu \geq 1000$ ) usually guarantees local equilibrium. With the assumption of local equilibrium, the fraction of occupied tramps $(\theta)$ is a function only of the lattice hydrogen concentration $\left(c_{L}\right)$ :

$$
\theta=\frac{K c_{L}}{1+K c_{L}} ; \quad K=\frac{k_{T}}{\rho},
$$

where $K$ is the equilibrium constant of the trapping reaction. The resulting differential equation is

$$
\frac{\partial C_{L}}{\partial t}+N \frac{\partial}{\partial t}\left(\frac{K c_{L}}{1+K c_{L}}\right)=D_{L} \frac{\partial^{2} c_{L}}{\partial x^{2}}
$$

If $K c_{L} \ll 1$ (weak traps, defined as those with $\theta \ll 1$ ), then (22) is simplified and can be analytically solved. The solution for the $\mathrm{H}$ flux $J_{\mathrm{H}}\left[\mathrm{mol} \mathrm{H} /\left(\mathrm{cm}^{2} \mathrm{~s}\right)\right]$ of the permeation buildup transient is

$$
J_{\mathrm{H}}=\frac{D_{\mathrm{app}} C_{0}}{L}\left(1+2 \sum_{n=1}^{\infty}(-1)^{n} \exp \left[-n^{2} \pi^{2} \frac{D_{\mathrm{app}} t}{L^{2}}\right]\right),
$$

where $c$ denotes the $\mathrm{H}$ concentration located either in NILS or in weak trap sites as defined in (22); $c_{0}$ specifies the concentration $c$ at a location just beneath the input surface of the permeation membrane. $D_{\text {app }}$ is the apparent diffusion coefficient which is also defined in (22):

$$
c=C_{L}+c_{W}=c_{L}\left(1+K_{W} N_{W}\right) ; \quad D_{\text {app }}=\frac{D_{L}}{1+K_{W} N_{W}} .
$$

The subindex $W$ in (22) is used to denote "weak" traps. According to Oriani [5], $c=c_{L}\left(1+K_{W} N_{W}\right)$. Particularly at the entry surface $c_{0}=c_{L 0}\left(1+K_{W} N_{W}\right)$. Equation (21) shows that the weak traps do not cause a distortion in the shape of the permeation transient but just a change in the time scale with respect to a trap free material with $D_{\text {app }}=D_{L}$. It is worth noting that, according to (22), different combinations of $K_{W} N_{W}$ would yield the same value of $D_{\text {app }}$ and consequently would produce indistinguishable permeation transients, as mentione in Section 3. This means that a single permeation transient does not allow assessing individual values of weak trap density and weak trap energy. The present treatment is developed in such way that the effect of the weak traps is only reflected in the value of $D_{\text {app }}$. In the presence of strong traps ( $\theta$ not negligible against unity), (20) has no analytical solution but must be solved by a numerical method. Before dealing with the numerical method itself, (20) is modified by taking into account that, according to (21), the ensemble of lattice and weak traps behaves as a simple diffusion system. Then, (20) is rewritten in terms of $D_{\text {app }}, c$, the density of strong traps $\left(N_{S}\right)$ and the equilibrium constant for the trapping reaction of the strong traps $K_{S}$ :

$$
\frac{\partial c}{\partial t}+N_{s} \frac{\partial}{\partial t}\left(\frac{K_{s} c_{L}}{1+K_{s} c_{L}}\right)=D_{\mathrm{app}} \frac{\partial^{2} c}{\partial x^{2}}
$$


In the above equation the effect of the weak trap density and energy is implicitly reflected in the value of $D_{\text {app }}$. Then, the only traps to be considered explicitly are the strong traps. Equation (23) expresses the central idea of the present method, which is the use of $D_{\text {app }}$ instead of $D_{L}$. and concentrates on the strong traps which are the ones that change the shape of the permeation transient. Now, according to (22), the lattice concentration $c_{L}$ is expressed in terms of $c$ :

$$
\frac{\partial c}{\partial t}+N_{s} \frac{\partial}{\partial t}\left(\frac{K^{\prime} c}{1+K^{\prime} c}\right)=D_{\text {app }} \frac{\partial^{2} c}{\partial x^{2}},
$$

where

$$
K^{\prime}=\frac{K_{s}}{1+K_{W} N_{W}} .
$$

The constant $K^{\prime}$ in (24) is related to $K_{S}$ by (25). $K_{s}$ is the true equilibrium constant which uses the lattice hydrogen concentration as the reference state. The numerical methods to solve the McNabb and Foster's equations presented previously (Thomas and Stern [3], Johnson and Lin [4], Ferris and Turnbull [6], Ramunni et al. [7]) use the lattice diffusion coefficient $D_{L}$. The present approach uses, instead of $D_{L}$, an apparent diffusion coefficient $D_{\text {app }}$ which contains the effect of the weak traps.

The advantages of this method are as follows.

(i) The focus is on the strong traps, about which the shape of the permeation transient contains valuable information.

(ii) $D_{\text {app }}$ may be orders of magnitude lower than $D_{L}$; therefore, the calculation speed is improved since the time steps are inversely proportional to the diffusion coefficient as it is shown below.

\section{Numerical Method}

To solve the differential equation (24) a finite difference method is used. The permeation membrane is divided in $m$ slices or volume elements of thickness $L / m$. Each volume element is identified by an index $j(1 \leq j \leq m)$. The extreme values $j=1$ and $j=m$ correspond to volume elements adjacent to the entry and exit side of the permeation membrane, respectively. The diffusion path is $L / m$ between adjacent slices and $L / 2 m$ for the entry and exit interfaces. The diffusion into the volume element is described by the right hand member of (24). The overall $\mathrm{H}$ concentration $\left(c_{T}\right)$ is defined as the sum of lattice, weakly trapped and strongly trapped hydrogen concentrations: $c_{T}=c_{L}+c_{W}+c_{S}=c+c_{S}$. The numerical calculation proceeds from an initial condition in a succession of cycles. Each cycle is composed of two steps. The first step of the method is to calculate $\Delta c_{T j}$ which is defined as the increase of the overall $\mathrm{H}$ concentration in each volume element $(j)$ by diffusion during a time interval $(\Delta t)$. $\Delta c_{T j}$, is calculated via the right hand member of (24), where the second order partial derivatives are approximated by finite increments, taking into account the different diffusion paths:

$$
\begin{gathered}
\Delta c_{T j}=D_{\mathrm{app}} \frac{\partial^{2} c}{\partial x^{2}} \Delta t, \\
\frac{\partial^{2} c}{\partial x^{2}} \simeq \frac{2 c_{0}+c_{2}-3 c_{1}}{(L / m)^{2}} \Delta t, \quad(j=1), \\
\frac{\partial^{2} c}{\partial x^{2}} \simeq \frac{c_{j-1}+c_{j+1}-c_{j}}{(L / m)^{2}} \Delta t, \quad(1<j<m), \\
\frac{\partial^{2} c}{\partial x^{2}} \simeq \frac{2 c_{m+1}+c_{m-1}-3 c_{m}}{(L / m)^{2}} \Delta t, \quad(j=m) .
\end{gathered}
$$

The second step is to achieve simultaneously the mass conservation and local equilibrium condition within each volume element. For only one type of strong traps, the following quadratic equation must be solved for $c_{\text {new: }}$ :

$$
c_{\text {old }}+\frac{N_{S} K^{\prime} c_{\text {old }}}{1+K^{\prime} c_{\text {old }}}+\Delta c_{T}=c_{\text {new }}+\frac{N_{S} K^{\prime} c_{\text {new }}}{1+K^{\prime} c_{\text {new }}},
$$

where the subindexes "old" and "new" refer to the values of $c$ before and after the time interval $\Delta t$ has elapsed. Once $c_{\text {new }}$ is calculated for each volume element, the $\mathrm{H}$ flux at the exit side of the permeation membrane $J_{\mathrm{H}}$ is calculated with Fick's law:

$$
J_{\mathrm{H}}=-D_{\text {app }} \frac{\partial c}{\partial x}(x=L) \simeq D_{\text {app }}\left(\frac{c_{j}-c_{j+1}}{L / 2 m}\right) .
$$

A usual technique to detect $\mathrm{H}$ at the exit side of the permeation membrane is the electrochemical method. In this method, the measured hydrogen current density $i_{\mathrm{H}}$ is related to $J_{\mathrm{H}}$ by the Faraday's constant $F$ :

$$
i_{\mathrm{H}}=\frac{J_{\mathrm{H}}}{F},
$$

provided that $\mathrm{H}$ concentration is expressed as $\mathrm{molH}$ per unit volume. After carrying out the former procedures, the time is increased in $\Delta t$ and the cycle is repeated from the first step. The successive values of $i_{\mathrm{H}}$ as a function of time constitute the "calculated" or "simulated" permeation transient. The time interval $\Delta t$ is chosen sufficiently low in order to keep the system stable. For this kind of diffusion systems $\Delta t$ is usually given by

$$
\Delta_{t}=\frac{L^{2}}{D_{\text {app }} m^{2}} r,
$$

where $r$ is the so-called mesh ratio [3]. For the present system, $r \leq 0.2$ guarantees numerical stability. Taking into account (30), (27) can be rewritten as:

$$
\begin{gathered}
\Delta c_{T j} \simeq r\left(2 c_{0}+c_{2}-3 c_{1}\right), \quad(j=1), \\
\Delta c_{T j} \simeq r\left(c_{j-1}+c_{j+1}-c_{j}\right), \quad(1<j<m), \\
\Delta c_{T j} \simeq r\left(2 c_{m+1}+c_{m-1}-3 c_{m}\right), \quad(j=m) .
\end{gathered}
$$

The method described above considers only one type of strong traps. However, it can be easily generalized to many 
types of traps with densities $N_{S 1}, N_{S 2}, \ldots$ and equilibrium constants $K_{S 1}, K_{S 2}, \ldots$ by rewriting (27):

$$
\begin{aligned}
c_{\text {old }} & +\frac{N_{S 1} K_{1}^{\prime} c_{\text {old }}}{1+K_{1}^{\prime} c_{\text {old }}}+\frac{N_{S 2} K_{2}^{\prime} c_{\text {old }}}{1+K_{1}^{\prime} c_{\text {old }}}+\cdots+\Delta c_{T} \\
& =c_{\text {new }}+\frac{N_{S 1} K_{1}^{\prime} c_{\text {new }}}{1+K_{1}^{\prime} c_{\text {new }}}+\frac{N_{S 2} K_{2}^{\prime} c_{\text {new }}}{1+K_{2}^{\prime} c_{\text {new }}}+\cdots
\end{aligned}
$$

In this case, (32) has no analytical solution and $c_{\text {new }}$ is obtained by a numerical method such as the NewtonRaphson method. For the present calculations, the values adopted for the mesh ratio and the number of volume elements are $r=0.2$ and $m=40$, respectively. The choice of $m=40$ guarantees sufficient accuracy of the numerical solution.

5.1. The Free Energy of Trapping. The free energy of trapping $\Delta G$ can be obtained from the thermodynamical relation:

$$
\Delta G^{0}=-R T \ln K
$$

where $R$ is the universal gas constant and $T$ is the absolute temperature. $\Delta G^{0}$ is the standard change of free energy for the trapping reaction. The reference state for hydrogen as solute in NILS is of the Henry's type (ideal dilute solution) with $c_{L}=1 \mathrm{~mol} \mathrm{~cm}^{-3}$. The reference state for trapped hydrogen corresponds to a trap occupancy $\theta=0.5$. In the rest of the present article, $\Delta G$ and "free energy of trapping" are used instead of $\Delta G^{0}$ and "standard change of free energy for the trapping reaction" for the sake of simplicity. Activity coefficients are assumed to be unity throughout this paper. For the case of the strong traps, it is useful to express their free energy of trapping $\Delta G_{S}$ as a function of the parameters $K^{\prime}$ and $D_{\text {app }}$ used for the calculation of the permeation transient as described above. This is accomplished by combining (22), (25) and (33):

$$
\Delta G_{S}=\Delta G^{\prime}-R T \ln \left(\frac{D_{L}}{D_{\text {app }}}\right) ; \quad \Delta G^{\prime}-R T \ln K^{\prime} .
$$

In fact, $\Delta G^{\prime}$ is the parameter handled by the software. In order to obtain $\Delta G_{S}, \Delta G^{\prime}$ must be corrected according to (34).

5.2. Rising and Degassing Transients. The present method is able to calculate either rising and degassing permeation transients. This is achieved by choosing the appropriate initial and boundary conditions. For the rising transient the initial condition is $c_{j}=0$ for $1<j<m$ and the boundary conditions are $c_{j}=c_{0}$ for $j=0$ and $c_{j}=0$ for $j=m+1$. For the degassing transient the initial condition is $c_{j}=c_{0}(m j+$ $1 / 2$ ) for $1 \leq j \leq m$ and the boundary conditions are $c_{j}=0$ for $j=0$ and $c_{j}=0$ for $j=m+1$. The concept of weak and strong traps has been defined only qualitatively in the former paragraphs, and a quantitative criterion to establish whether a trap is weak or strong has been investigated. For iron base alloys, it is expected that this criterion is essentially the value of the free energy of trapping $\Delta G$, but also the temperature and the input hydrogen activity can
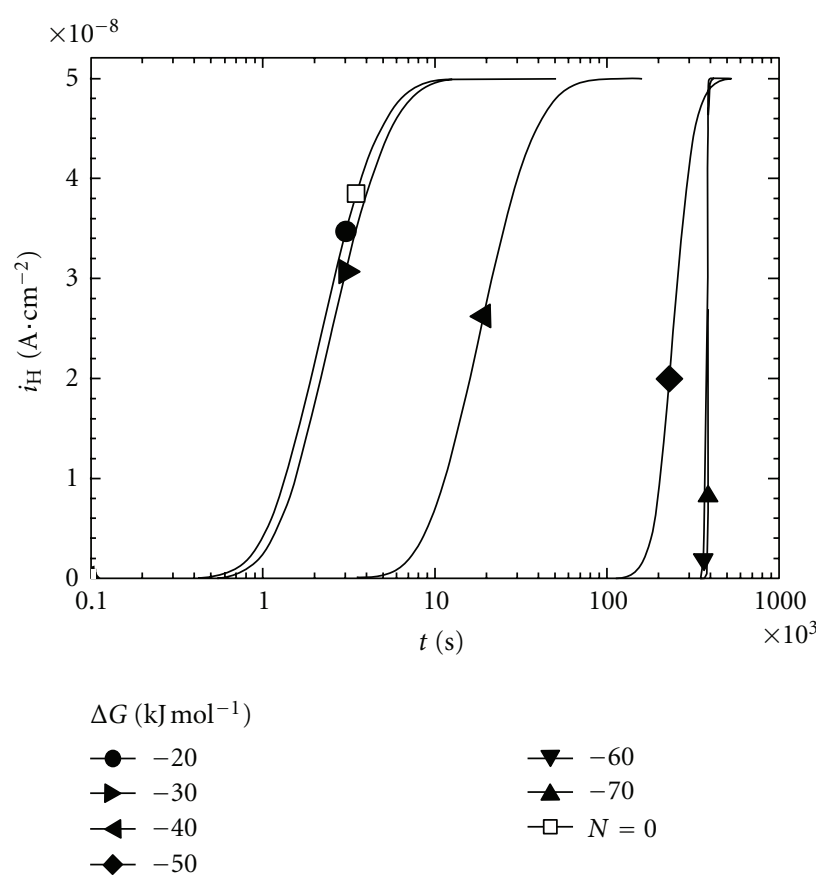

Figure 1: Effect of the free energy of trapping $\Delta G$ in the hydrogen permeation transients calculated by a finite difference method. Empty symbol: trap free lattice $\left(N=0,(21)\right.$ with $\left.D=D_{L}\right)$. Filled symbols: lattice with traps $\left(N=10^{-6} \mathrm{~mol} \mathrm{~cm}^{-3}\right)$ with different values of $\Delta G$.

play a secondary role. In the present work the effect of $|\Delta G|$ has been investigated. The input hydrogen activity assumed here is that of typical gas phase charging experiments (hydrogen partial pressure $=0.1 \mathrm{MPa}$ ), and the temperature is that of typical electrochemical permeation experiments (303 K). The parameter $i_{\mathrm{H}_{\mathrm{ss}}}$ is obtained from the permeation coefficient of iron [9]. The assumed diffusion coefficient is $10^{-5} \mathrm{~cm}^{2} \mathrm{~s}^{-1}$, typical of a trap free material [9]. For a first series of runs $\Delta G$ is varied from 20 to $50 \mathrm{~kJ} / \mathrm{mol}$. The value of $K$ is obtained with (33), and the value of $N$ is chosen in order to keep the value of $K \times N$ fixed, that is, $K \times N=20$. Figure 1 shows the results of a first series of calculated rising permeation transients. The calculated hydrogen permeation flux $J_{\mathrm{H}}$ has been converted to its equivalent current density $i_{\mathrm{H}}=F \times J_{\mathrm{H}}$, where $F=96485 \mathrm{~A} \mathrm{~s} / \mathrm{mol}$ is the Faraday constant (electrochemical detection method). The parameters are chosen as those typical of a permeation experiment on a low alloy steel under gas phase charging: $L=004 \mathrm{~cm} ; D_{L}=$ $10^{-5} \mathrm{~cm}^{2} \mathrm{~s}^{-1}$; trap density $N=10^{-6} \mathrm{~mol} / \mathrm{cm}^{3}$; steady state hydrogen permeation current $i_{\mathrm{H}, \mathrm{ss}}=5 \times 10^{-8} \mathrm{~A} / \mathrm{cm}^{2}$ and temperature $30^{\circ} \mathrm{C}$. The free energy of trapping $\Delta G$ is changed from -20 to $-70 \mathrm{~kJ} / \mathrm{mol}$. Figure 1 also shows the diffusion transient for a material free of traps $(N=0)$, which is calculated by using the analytical solution of the Fick's law (11) and the lattice diffusion coefficient $D_{L}$. The transients that correspond to low values of $|\Delta G|$ from 20 to $40 \mathrm{~kJ} / \mathrm{mol}$, present the same shape and slope when the time scale is logarithmic. The effect of increasing $|\Delta G|$ is the displacement of the transient to the right. This is in agreement with 
Oriani local equilibrium theory for low trap occupancy, Fick's equation can be solved by replacing the lattice diffusion coefficient $D_{L}$ by an apparent diffusion coefficient $D_{\text {app }}(10)$. Then, as long as the trap occupancy is low, the effect of the traps is just to cause a delay in the permeation transient through an increase of the time by the factor $1+K N$, which is seen as a shift along the logarithmic time scale of the Figure 1. As $|\Delta G|$ increases beyond $40 \mathrm{~kJ} / \mathrm{mol}$ the slope of the permeation transient (logarithmic time scale) increases. For trapping energies equal or higher than $70 \mathrm{~kJ} / \mathrm{mol}$ the rise in the permeation flux is abrupt. $|\Delta G|$ values higher than $70 \mathrm{~kJ} / \mathrm{mol}$ yield permeation transients which can not be visually distinguished in Figure 2. This is in qualitative agreement with the prediction of $\Delta G_{\max }$ in Table 1 . The latter situation corresponds to the lattice diffusion of hydrogen in the presence of irreversible traps. The hydrogen front moves across the membrane thickness in a quasi-steady state process according to a parabolic law:

$$
t(x)=\frac{N x^{2}}{2 D_{L} c_{0}},
$$

where $x$ is the distance advanced by the diffusion front, measured from the input side of the permeation membrane. At the breakthrough time $t_{b}$ the diffusion front reaches the exit surface $(x=L)$, then:

$$
t_{b}=\frac{N L F}{2 i_{\mathrm{H}, \mathrm{ss}}},
$$

where $i_{\mathrm{H}, \mathrm{ss}}=\left(D_{L} c_{0} / L\right) F$ is the steady state hydrogen permeation current density. For the present set of parameters, $t_{b}$ calculated from (36) is $3.86 \times 10^{5} \mathrm{~s}$, in agreement with Figure 1. The expected permeation behavior for low values of $|\Delta G|$ in the range $30<|\Delta G| / \mathrm{kJ} \mathrm{mol}^{-1}<50$ is showed with more detail in Figure 2, by setting appropriate values of the parameters. The equilibrium constant $K$ is calculated with (5) and the trap density $N$ is chosen to keep the product $K \times N$ constant. Figure 2 shows (a) the analytical solution of the Fick's equation using $D_{\text {app }}$ calculated with (10) (Fick's transient); (b) the simulated permeation transients with different free energies of trapping $\Delta G$. The simulated transients with $|\Delta G|<35 \mathrm{~kJ} / \mathrm{mol}$ are practically coincident with the Fick's transient. This sets a practical limit to the validity of (10). The corresponding theoretical value from Table 1 is $33.7 \mathrm{~kJ} / \mathrm{mol}$. Contrarily, the simulated transients with $|\Delta G|>35 \mathrm{~kJ} / \mathrm{mol}$ are faster than the Fick's transient and present a different shape, that is, a steeper rise as $|\Delta G|$ increases. This feature allows the determination of $\Delta G$ in this range of values through a numerical fitting procedure. The effect of $\Delta G$ on the simulated hydrogen permeation degassing transients is also studied. The simulated degassing transient is calculated by the finite difference method, by changing the initial and boundary conditions of the system: zero hydrogen concentration is imposed both on the input and exit surfaces of the permeation membrane, and an initial linear lattice concentration profile is set within the membrane. The number of layers, membrane thickness, and other parameters are the same as those used for the simulation of the rising transients. Figure 3 shows the behavior of the

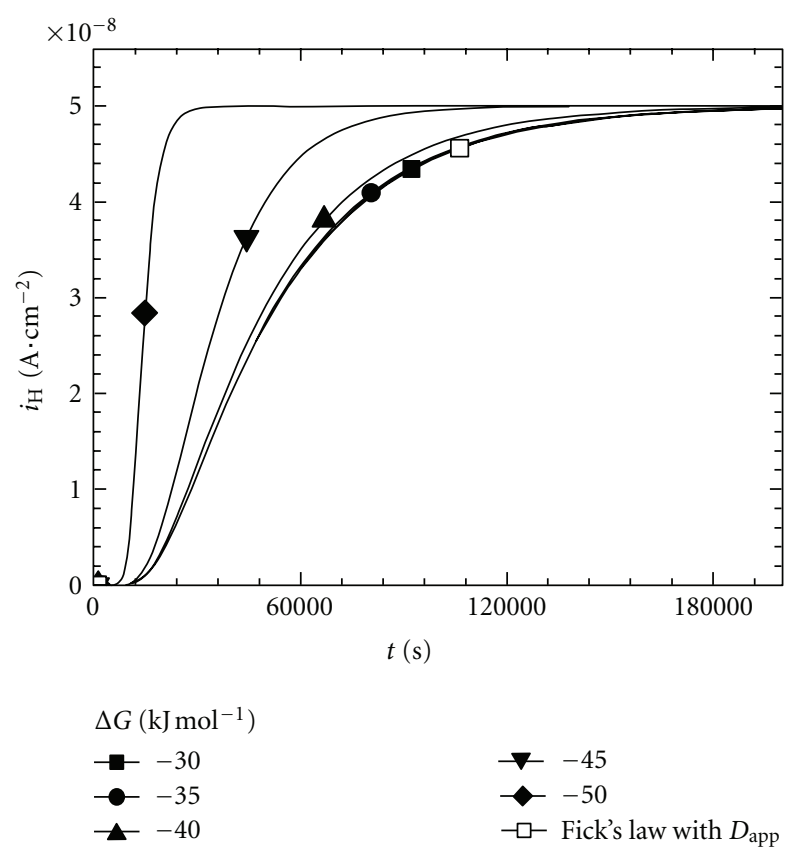

FIGURE 2: Hydrogen permeation transients calculated by a finite difference method to investigate the deviation from Oriani's equation (10). $K \times N=20$. Filled symbols: different calculated $K$ values from different free energy of trapping $\Delta G$. Empty symbol: analytical solution of the Fick's law (21), with $D=D_{\text {app }}$ from (10).

degassing transients, plotted versus a logarithmic time scale, when the trap density $N$ is fixed, and different values of the free energy of trapping in the range $20<|\Delta G| / \mathrm{kJ} \mathrm{mol}^{-1}<$ 80 are considered. For $|\Delta G|<40 \mathrm{~kJ} / \mathrm{mol}$ the shape of the degassing transient is not altered with respect to the transient calculated with (11) using the diffusion coefficient set by (10) (Oriani's domain). For $|\Delta G| \leq 40 \mathrm{~kJ} / \mathrm{mol}$, the effect of increasing values of $|\Delta G|$ is just to shift the degassing transient to the right, showing that the low trap occupancy hypothesis assumed in (10) is valid. For $|\Delta G|>40 \mathrm{~kJ} / \mathrm{mol}$ initially, the transient seems to be fast, but later a stage appears where the permeation flux decays slowly. This can be roughly interpreted as follows. In the first fast stage, most of the lattice hydrogen evolves; in the second slow stage the high energy traps, most of them filled, lose hydrogen via lattice diffusion driven by a low lattice concentration gradient. Figure 3 shows that values of $|\Delta G|$ as high as $70 \mathrm{~kJ} / \mathrm{mol}$ could theoretically be assessed through the analysis of degassing transients. Figure 4 shows more details on the behavior of the degassing transients in the intermediate energy range. When the product $K \times N$ is kept constant, the different degassing transients agree with that calculated with (10) and (11). The departure from this coincidence begins for $|\Delta G|$ values equal or higher than $40 \mathrm{~kJ} / \mathrm{mol}$. These observations are similar to those for the permeation rising transients in Figure 2.

5.3. Fitting Procedure. Besides calculating the permeation rising or degassing transient, the developed software allows the possibility to repeat the calculation with varied parameters in order to fit the calculated transient to experimental 

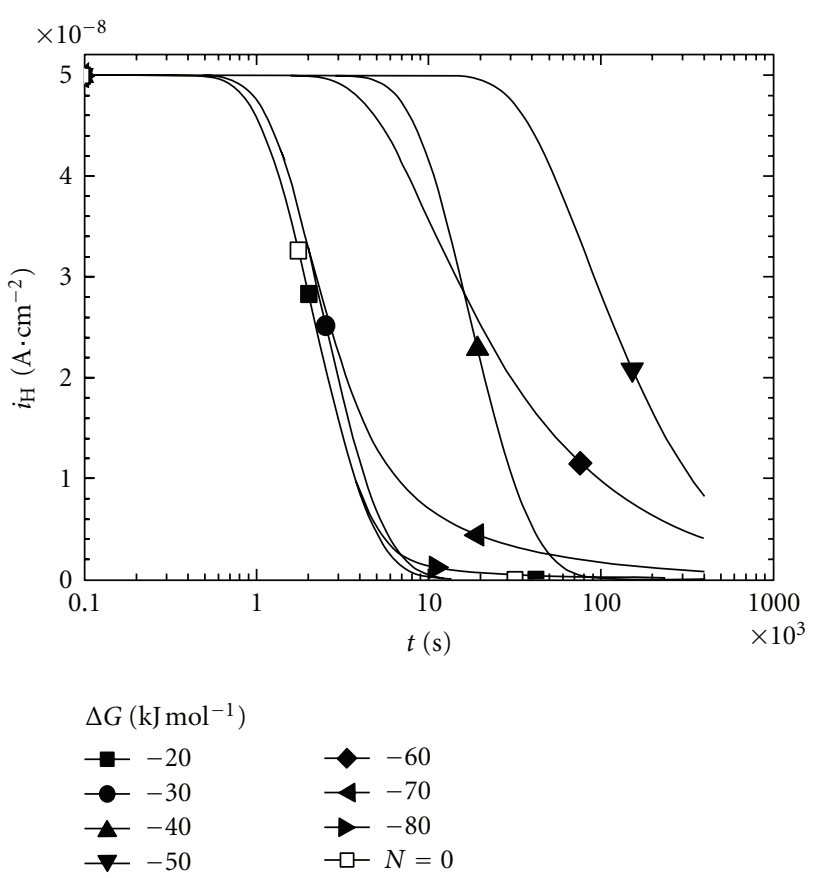

FIGURE 3: Effect of the free energy of trapping $\Delta G$ in the hydrogen degassing transients calculated by a finite difference method. Empty symbol: trap free lattice $\left(N=0,(21)\right.$ with $\left.D=D_{L}\right)$. Filled symbols: lattice with traps $\left(N=10^{-6} \mathrm{~mol} \mathrm{~cm}^{-3}\right)$ with different values of $\Delta G$.

data. The initial list of parameters $\left(L, T, i_{\mathrm{H}_{\mathrm{ss}}}, D_{\mathrm{app}}, \Delta G\right.$, $K)$ is supplied by the user. The parameter $i_{\mathrm{H}_{\mathrm{ss}}}$ is the steady state hydrogen current density. All parameters of this list except $L$ and $T$ can be selected to vary. The software used in the present work admits as many as two types of strong traps. The fitting criterion is the minimization of the mean quadratic difference between calculated and experimental points, defined as

$$
\text { Deviation }=\frac{1}{i_{\mathrm{H}_{\mathrm{ss}}}} \sqrt{\frac{1}{n_{\exp }} \sum_{k=1}^{n_{\text {exp }}}\left(i_{\mathrm{H}, \mathrm{cal}, k}-i_{\mathrm{H}, \exp , k}\right)^{2} \cdot 100},
$$

where $n_{\exp }$ is the number of experimental points; $i_{\mathrm{H}, \mathrm{calc}, j}$ and $i_{\mathrm{H}, \exp , j}$ are the calculated and experimental hydrogen current density, respectively.

\section{Summary.}

(i) The present method serves to analyze experimental hydrogen permeation rising or degassing transients via a least squares fitting procedure.

(ii) It is based on the McNabb and Foster's model of saturable traps with the Oriani's assumption of local equilibrium.

(iii) Domains of "weak trap", "strong trap", and strong trap with "diffusion front" behavior have been established for the present experimental conditions as a function of the free energy of trapping $\Delta G$.

(iv) The effect of the weak traps is reflected in the value of the apparent diffusion coefficient $D_{\text {app}}$, which is obtained as a fitting parameter.

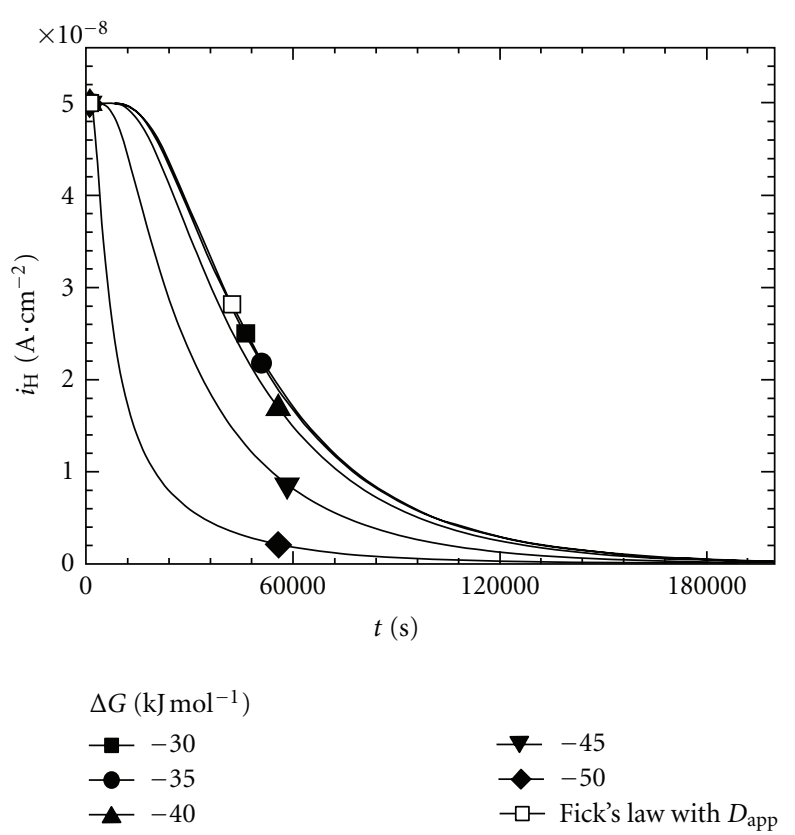

FIGURE 4: Hydrogen degassing transients calculated by a finite difference method to investigate the deviation from Oriani's equation (10). $K \times N=20$. Filled symbols: different calculated $K$ values from different free energies of trapping $\Delta G$. Empty symbol: analytical solution of the Fick's law (21), with $D=D_{\text {app }}$ from (10).

(v) Strong traps are characterized by a trap density $N$ and a free energy of trapping $\Delta G . N$ is directly obtained as a fitting parameter; $\Delta G$ is obtained from the fitting parameter $\Delta G^{\prime}$ by an equation that takes into account the change of the reference state implied by the use $D_{\text {app }}$ instead of $D_{L}$.

(vi) In the "diffusion front" domain, $N$ can be accurately determined but not $\Delta G$.

In the present work, up to one type of trap has been characterized. However, the developed method allows to increase that number if necessary.

\section{Applications: API 5L X60 Steel, As-Received (AR) Condition}

We check our model performing electrochemical permeation tests on an API 5L X60 steel taken from a seamless pipe manufactured in Argentina. This steel is employed as pipes conductors in the natural gas and petroleum industries and are characterized by their good properties of mechanical strength, weldability, and fracture toughness. The chemical composition is shown in Table 2. The material is submitted to experimental permeation tests as directly received from manufacturer, named the as received condition (AR).

In a previous work [11] it is reported the microstructural characterization and the study of nonmetallic inclusions of this material in the as received (AR) condition. The metallographic observation indicated that the API 5L X60 microstructure contains equiaxed ferrite grains and carbides 
TABLE 2: Chemical composition of the API 5L X60 steel (weight \%).

\begin{tabular}{lccccccccccccc}
\hline $\mathrm{C}$ & $\mathrm{Mn}$ & $\mathrm{Si}$ & $\mathrm{Cr}$ & $\mathrm{Mo}$ & $\mathrm{V}$ & $\mathrm{Cu}$ & $\mathrm{Ni}$ & $\mathrm{Al}$ & $\mathrm{P}$ & $\mathrm{S}$ & $\mathrm{Ti}$ & $\mathrm{Nb}$ \\
\hline 0.14 & 1.04 & 0.25 & 0.07 & 0.08 & 0.03 & 0.03 & 0.05 & 0.026 & 0.014 & 0.011 & 0.015 & 0.001 \\
\hline
\end{tabular}

mainly located in the grain boundaries, which is typical of tempered martensite; the grain size average as $4 \mu \mathrm{m}$, and the mean size of the cementite particles is ca. $0.3 \mu \mathrm{m}$. Analysis of the local chemical composition by Energy Dispersive $\mathrm{X}$ ray Spectroscopy microanalysis (EDAX) revealed few nonmetallic inclusions containing varying amounts of $\mathrm{Ca}, \mathrm{S}$, $\mathrm{Al}$, and Ti. From this material a parallelepiped of ca. $3.5 \mathrm{~mm}$ thickness is constructed. A disk with a thickness of $1.57 \mathrm{~mm}$ and a diameter of $35 \mathrm{~mm}$ is used in the electrochemical permeation tests as the permeation membrane. Prior to the tests, both disk faces (entry and exit surface of the permeation membrane) are ground with $\mathrm{SiC}$ up to $\# 600$ grit and then electropolished in a mixture of $10 \mathrm{HClO}_{4}$ and 90 butyl cellosolve at a temperature below $5^{\circ} \mathrm{C}$ under a potential difference of $36 \mathrm{~V}$. The objective of electropolishing is to eliminate a layer of $10 \mu \mathrm{m}$ of material from each membrane face, which is the estimated thickness of the deformed layer created by mechanical polishing. Then, a palladium film about $10 \mathrm{~nm}$ thick is electrochemically deposited on both membrane entry and exit surfaces. The deposition cell is equipped with a $\mathrm{Pd} / \mathrm{PdH}$ reference electrode and a Pt counter electrode, and it is filled with ca. $300 \mathrm{~mL}$ of electrolyte in aqueous solution of $0.1 \mathrm{M}$ $\mathrm{NaOH}$. The electrolyte is continuously deaerated by nitrogen bubbling. Prior to the deposition, the permeation membrane is etched in $\mathrm{HCl} 50$, rinsed with water, and immediately subjected to cathodic polarization in the electrochemical cell at $0.1 \mathrm{~mA} / \mathrm{cm}^{2}$ current density. The cathodic treatment continued until the typical potential of $\mathrm{H}_{2}$ evolution on the steel $(-0.970 / \mathrm{mV}$ versus normal hydrogen electrode, NHE) is reached. The deposition is started by introducing in the cell $7 \mathrm{~mL}$ of an aqueous solution of $\left[\mathrm{Pd}\left(\mathrm{NO}_{2}\right)_{4}\right] \mathrm{Na}_{2}$ which contains $0.004 \mathrm{~g} / \mathrm{mL}$ of Pd while keeping the current density at $0.1 \mathrm{~mA} / \mathrm{cm}^{2}$. The deposition time is around 40 minutes. Finally. the Pd coated membrane is degassed in an oven at $110^{\circ} \mathrm{C}$ during 16 hours. The $\mathrm{H}$ permeation tests are carried out using gas phase charging and electrochemical detection as showed in Figure 5.

Gas phase charging ensures a constant and reliable chemical potential of hydrogen at the input surface [12]. The electrochemical detection technique is according to Devanathan and Stachurski [13] in an experimental arrangement similar to that outlined in [14]. This arrangement consists of two compartments (input cell and detection cell) separated by the steel permeation membrane which has an exposed area of $1.9 \mathrm{~cm}^{2}$ on both their entry and exit surfaces. Gas phase charging is achieved by circulating $\mathrm{H}_{2}(\mathrm{~g})$ (99.999) at = 1 bar through the input cell. The Pd film at the entry surface allows reaching equilibrium between the gas phase and the metal phase according to Sieverts law [12]. The detection cell includes a $\mathrm{Pd} / \mathrm{PdH}$ reference electrode and a platinum wire as counter electrode. It contained a $0.1 \mathrm{M} \mathrm{NaOH}$ solution which is continuously deaerated

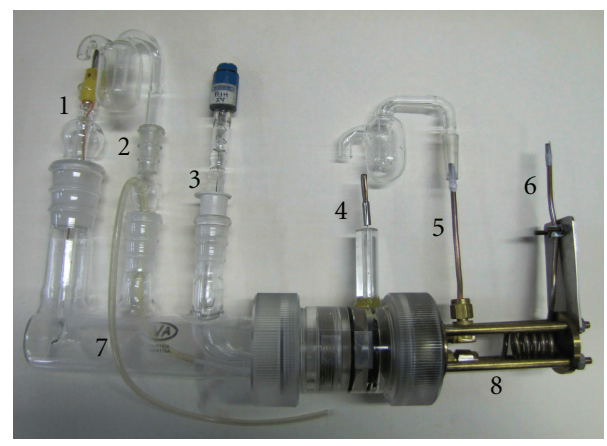

FIgURE 5: Experimental permeation cell. The scheme shows the components of the equipment. Numbers are (1). Platinum electrode (Pt), (2). Input and output of nitrogen $\left(\mathrm{N}_{2}\right),(3)$. Reference electrode $\left(\mathrm{Pd} / \mathrm{H}_{2}\right),(4)$. Permeation membrane (sample) (5). Hydrogen flux output (Rising transient), nor air output (decreasing transient), (6). Hydrogen flux input (Rising transient), nor air input (decreasing transient), (7). Electrochemical detection cell, and 8. Generation cell.

with nitrogen. The exit surface of the metallic membrane is polarized at $+200 \mathrm{mV}$ with respect to NHE. The $\mathrm{H}$ flux at the exit surface is measured as its faradic equivalent $i_{\mathrm{H}}\left[\mathrm{A} / \mathrm{cm}^{2}\right]=i-i_{\mathrm{bg}}$, where $i_{\mathrm{bg}}$ is a background current density, mainly due to residual corrosion of the steel. The $i_{\mathrm{H}}$ values are recorded as a function of time $t[\mathrm{~s}]$. Permeation buildup or degassing transients are obtained by circulating either $\mathrm{H}_{2}(\mathrm{~g})$ or purified air, respectively, through the input cell. The tests are performed consecutively at $30^{\circ} \mathrm{C}, 50^{\circ} \mathrm{C}$, and $70^{\circ} \mathrm{C}$. At $30^{\circ} \mathrm{C}$, two successive sequences of permeation buildup and degassing transients are performed in order to detect the possible presence of high-energy traps. The values of the diffusion and trapping parameters are obtained by fitting calculated hydrogen permeation transients to the experimental transients. Unlike the approach by Ramunni et al. [7], the model and software employed in the present work to calculate the permeation transients is restricted to the case of local equilibrium. Hydrogen traps are classified according to their free energy of trapping $\Delta G$ in weak traps with $|\Delta G|<$ $35 \mathrm{~kJ} / \mathrm{mol}$ and strong traps with $-70<|\Delta G| \mathrm{kJ} \mathrm{mol}^{-1}<-35$. The effect of the weak traps is reflected in the value of $D_{\text {app }}$. For the representation of the experimental and calculated permeation transients, the net $\mathrm{H}$ current density $\left(i_{\mathrm{H}}\right)$ and the time $(t)$ are normalized with respect the membrane thickness $(L)$, that is, $i_{\mathrm{H}} \times L$ versus $t / L^{2}$. In presents calculations it is considered that the values of $N$ and $\Delta G$ should be independent of the test temperature, because the traps, that is, the nature and density of the metallurgical defects, are not expected to change at the test temperatures (30 to $70^{\circ} \mathrm{C}$ ). Thus, the best set of fitting parameters is selected by attempting a compromise between the goodness of the fit and the physical consistency of the results. Instead of directly 
TABLE 3: Calculated diffusion and trapping parameters from the permeation rising transients for the API 5L X60 AR steel condition.

\begin{tabular}{|c|c|c|c|c|c|}
\hline \multicolumn{6}{|c|}{ Rising transient } \\
\hline Temperature & $D_{\text {app }}$ & $i_{\mathrm{ss}}$ & $\Delta G$ & $N$ & Deviation \\
\hline${ }^{\circ} \mathrm{C}$ & $\mathrm{cm}^{2} / \mathrm{s}$ & $\mathrm{A} / \mathrm{cm}^{2}$ & $\mathrm{KJ} / \mathrm{mol}$ & $\mathrm{mol} \mathrm{H} / \mathrm{cm}^{3}$ & $\%$ \\
\hline 30 & $4.06 \times 10^{-6}$ & $1.28 \times 10^{-7}$ & -56.4 & $1.42 \times 10^{-8}$ & 1.18 \\
\hline 30 & $4.27 \times 10^{-6}$ & $1.28 \times 10^{-7}$ & -56.3 & $1.45 \times 10^{-8}$ & 1.26 \\
\hline 50 & $7.53 \times 10^{-6}$ & $3.13 \times 10^{-7}$ & -60.9 & $4.24 \times 10^{-9}$ & 0.87 \\
\hline 70 & $1.73 \times 10^{-5}$ & $6.12 \times 10^{-7}$ & -62.4 & $3.08 \times 10^{-9}$ & 0.58 \\
\hline
\end{tabular}

TABLE 4: Calculated diffusion and trapping parameters from the permeation degassing transients for the API 5L X60 AR steel condition.

\begin{tabular}{|c|c|c|c|c|c|}
\hline \multicolumn{6}{|c|}{ Degassing transient } \\
\hline Temperature & $D_{\text {app }}$ & $i_{\mathrm{ss}}$ & $\Delta G$ & $N$ & Deviation \\
\hline${ }^{\circ} \mathrm{C}$ & $\mathrm{cm}^{2} / \mathrm{s}$ & $\mathrm{A} / \mathrm{cm}^{2}$ & $\mathrm{KJ} / \mathrm{mol}$ & $\mathrm{mol} \mathrm{H} / \mathrm{cm}^{3}$ & $\%$ \\
\hline 30 & $4.27 \times 10^{-6}$ & $1.28 \times 10^{-7}$ & -56.3 & $1.45 \times 10^{-8}$ & 1.76 \\
\hline 30 & $4.27 \times 10^{-6}$ & $1.28 \times 10^{-7}$ & -56.3 & $1.45 \times 10^{-8}$ & 2.23 \\
\hline 50 & $7.53 \times 10^{-6}$ & $3.13 \times 10^{-7}$ & -60.9 & $4.24 \times 10^{-9}$ & 2.44 \\
\hline 70 & $1.73 \times 10^{-5}$ & $6.12 \times 10^{-7}$ & -62.4 & $3.08 \times 10^{-9}$ & 1.62 \\
\hline
\end{tabular}

using the optimal set of parameters (from the point of view of the mean quadratic deviation) for each test temperature, these values are varied slightly in order to achieve a unique value of trap density, independent of the test temperature. In this way, the deviation between calculated and experimental data increased slightly, up to 0.1 in \% units.

For the as received-API 5L X60 condition we assume only one kind of trapping site, and we demonstrate that it is enough to achieve an excellent fit of the calculated transient to the experimental one. This means that in (1) we consider $Q=1$ and only the term $N_{i} \theta_{i}=N_{1} \theta_{1}$ appears. In Table 3, we present the fitted parameters: $D, \Delta G$, and $N$, at different temperatures for the rising transients. Then, each set of fitted parameters is used to simulate the permeation degassing transients at the corresponding temperature.

At $30^{\circ} \mathrm{C}$ the 2 nd rising transient data is employed. The coincidence between the simulated and experimental degassing transients is satisfactory, as showed by the corresponding error values in Table 4. Fitted transient deviation (last column of Tables 3 and 4 is calculated as a mean quadratic deviation, referred to the steady state flux from (37)). In Figures 6, 7, and 8, the experimental rising transients are represented, together with the theoretical ones identified as fitted curve for the API 5L X60 steel, in the AR state at $30^{\circ} \mathrm{C}, 50^{\circ} \mathrm{C}$, and $70^{\circ} \mathrm{C}$. The selected set of parameters for the AR condition obtained from the buildup transients are reported in Tables 3 and 4 respectively. Tables 3 and 4 , also present the mean quadratic deviation (expressed as percentage with respect to the steady state current density) of the fitted transients. The agreement between fit and experimental transient is very good. It must be pointed out that the diffusion coefficient $D$ is not fixed as in [7]. Instead, it is allowed to vary in order to obtain the best fit. Therefore, the parameter $D$ should not be regarded as the lattice diffusion coefficient but to an apparent diffusion coefficient $D_{\text {app }}$ which contains the effect of weak trapping sites, as will be discussed below. From Tables 3 and 4 , it is

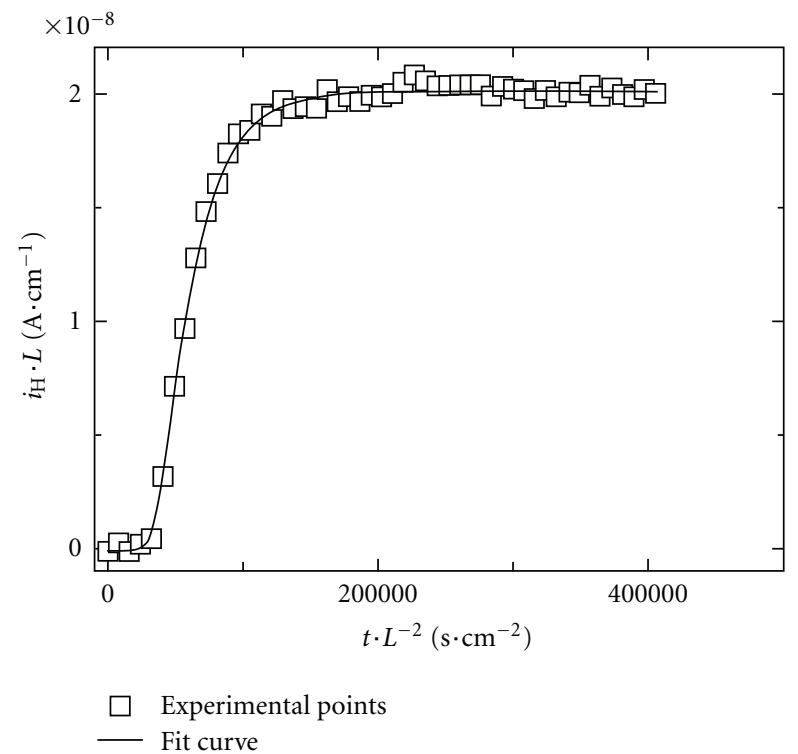

Figure 6: Rising permeation transient at $30^{\circ} \mathrm{C}$ for the API 5L X60 steel in the as received (AR) condition.

observed that the trap density $N$, used as a fitting parameter, is reduced as the test temperature $T$ increases. Contrar to what expected, the fitted values of $N$ and $\Delta G$ depend on temperature. The value of $N$ at $70^{\circ} \mathrm{C}$ is approximately 5 times lower than that at $30^{\circ} \mathrm{C}$. The absolute $\Delta G$ values are less sensitive to $T$, showing a moderate increase of $\Delta G$ with increasing $T$. The observed dependence of $N$ and $\Delta G$ with $T$ should be investigated with more detail in future works. Concerning the experiments at $30^{\circ} \mathrm{C}$, there is no significative difference between the 1 st and 2 nd rising permeation transients, revealing a negligible density of high energy or irreversible traps. At this point, it is important to analyze the influence of weak traps presents in the studied material. Our software considers $D$ as a fitting parameter, 


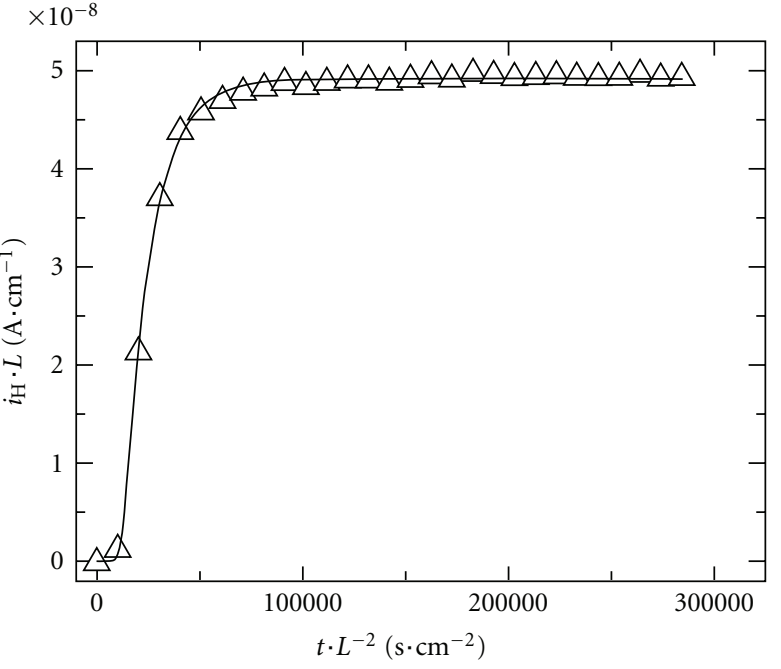

$\triangle$ Experimental points
Fit curve

Figure 7: Rising permeation transient at $50^{\circ} \mathrm{C}$ for the API 5L X60 steel in the as received (AR) condition.

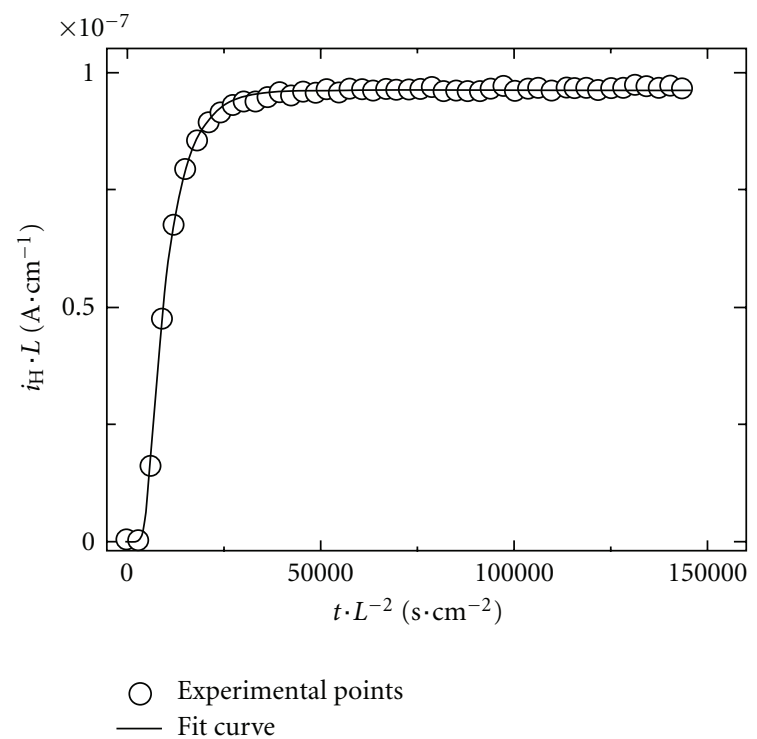

Figure 8: Rising permeation transient at $70^{\circ} \mathrm{C}$ for the API 5L X60 steel in the as received (AR) condition.

which is allowed to vary with the purpose of diminishing the error of the fit. Then, $D$ is an apparent diffusion coefficient $D_{\text {app }}$ corresponding to a crystalline lattice subject to the effects of weak traps, in concordance with (10). On the other hand, the coefficient $D_{L}\left[\mathrm{~cm}^{2} / \mathrm{s}\right]=5.12 \times$ $10^{-4} \exp \left(-4.15 \mathrm{~kJ} \mathrm{~mol}^{-1} / R T\right)$ [9] corresponds to a lattice without defects, through which hydrogen flows without being trapped, implying $D_{\text {app }}<D_{L}$. The use of $D_{\text {app }}$ instead of $D_{L}$ by the software necessarily implies that the hydrogen concentration handled by the software is not the lattice concentration $c$ but an overall concentration $c_{L}+w$ (lattice more weak traps). Assuming the validity of (10), it can

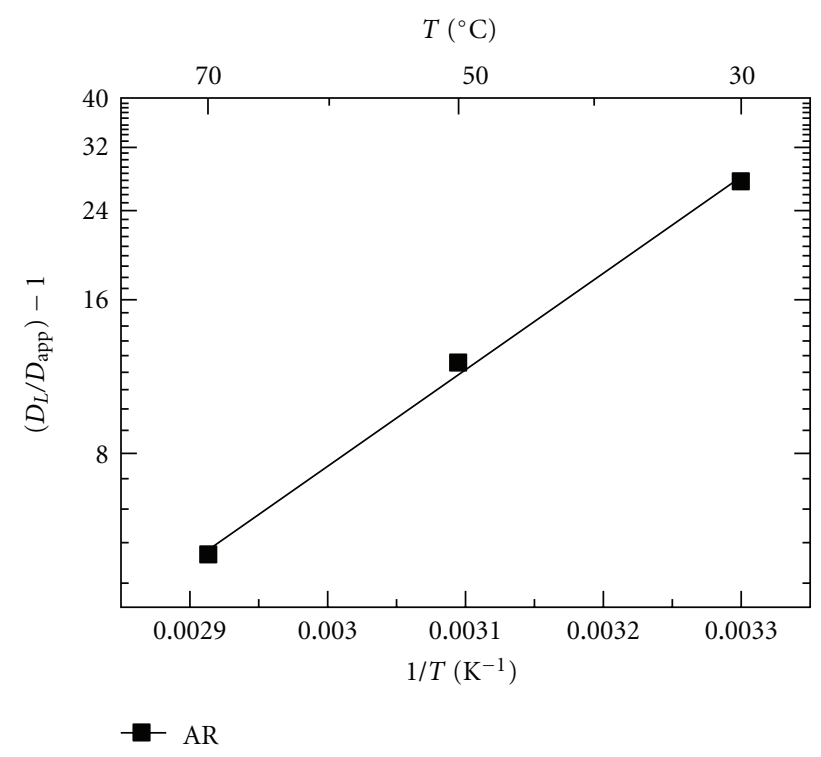

FIGURE 9: Arrhenius-type plot from rising transients at $30^{\circ} \mathrm{C}, 50^{\circ} \mathrm{C}$, and $70^{\circ} \mathrm{C}$ for the API 5L X60 steel in the as received (AR) condition.

be demonstrated that both concentrations are related by $c_{L}+w / c=\left(1+K_{w} N_{w}\right)=D_{L} / D_{\text {app }}$. Then, a correction is needed to refer the parameter $\Delta G^{\prime}$ yielded by the software to the lattice concentration. This correction is $\Delta G=\Delta G^{\prime}+$ $R T \ln \left(D_{\text {app }} / D_{L}\right)$. The $\Delta G$ values showed in Tables 3 and 4 are obtained by this procedure. As a consequence of the discussion presented above, the parameters $\Delta G$ and $N$ in 3 and 4 obtained from the fit reflect the influence of high energy traps but not of weak traps: the latter are implicit in $D_{\text {app }}$. The characterization of the weak traps in the present material necessarily implies an analysis of the dependence of $D_{\text {app }}$ on temperature, that is, $D_{\text {app }}(T)$. From (5) an expression can be obtained for the equilibrium constant $K_{\text {weak }}$ as:

$$
\ln K_{\text {weak }}=\ln \left(\frac{D_{L}}{D}-1\right)-\ln N_{\text {weak }}
$$

In Figure 9 we represent the parameter $\left(D_{L} / D_{\text {app }}-1\right)$ in an Arrhenius type plot, with $D_{\text {app }}$ being the fitted values of Table 3. We calculate the trapping enthalpy from the thermodynamical expression $d \ln K_{\text {weak }} / d T=H_{\text {weak }} / R T^{2}$, by linear regression of the corresponding data in Figure 9. Following this procedure we obtain $\Delta H_{\text {weak }}=28.1 \mathrm{~kJ} / \mathrm{mol}$. Assuming that the entropy of trapping $\Delta S_{\text {weak }}$ is negligible, then $\Delta G_{\text {weak }} \Delta H_{\text {weak }}$ and from the linear regression ordinate, we obtain a density traps of $N_{\text {weak }}=3.32 \times 10^{-4} \mathrm{~mol} / \mathrm{cm}^{3}$. The value of $\Delta G_{\text {weak }}$ represents the mean free energy of trapping of the weak or low occupancy traps; it lies within the Oriani domain according to the discussion of Section 4 .

\section{Validation of the Present Method}

Typical permeation transients calculated with the present method (which assumes local equilibrium) are also obtained by means of a software presented previously by Ramunni 


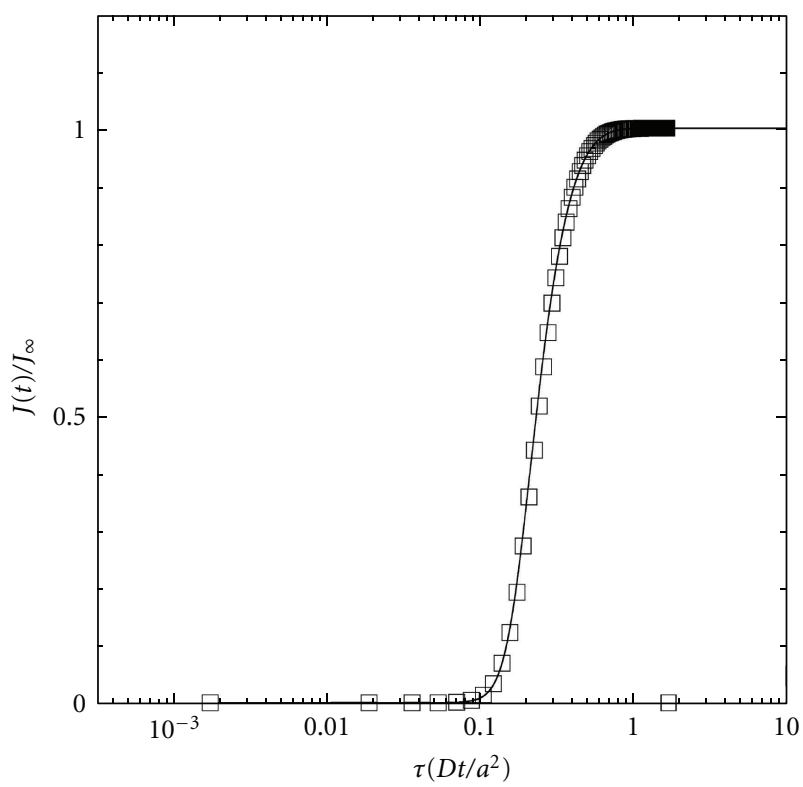

Figure 10: Comparison between the theoretical transients of the API 5L X60 steel in the AR condition at $30^{\circ} \mathrm{C}$, calculated with the present software (empty squares) and with the software of [7] (filled line). $J_{\infty}$ is the hydrogen flux in the steady state, $D=D_{\text {app }}$ and $a=$ $0.157 \mathrm{~cm}$.

et al. [7]. This software does not assume local equilibrium and directly solves the McNabb and Foster's equation (18). The parameters $\lambda, \rho$, and $\mu$ for the software of [7] are obtained through replacement of the parameters $D_{L}, K$, and $N$ in (18) and (19) by the present parameters $D_{\text {app }}$, $K^{\prime}$, and $N_{S}$ and assuming an arbitrary value for $\mu$. The software in [7], which directly solves the coupled McNabb and Foster differential equations, is as follows: we calculated the dimensionless parameters $\lambda=N k L^{2} / D, \rho=c_{0} / N$, and $\mu=p L^{2} / D$, as defined in [7]. The values of $N$ and $D$ are, respectively, the fitting parameters $N$ and $D_{\text {app }}$ for the API 5L X60-(AR) sample that are presented in Table 3. The parameter $\mu$ is above a certain critical value in order to fulfill the local equilibrium condition and is changed arbitrarily in order to establish such value. Once the $\mu$ value is fixed, the kinetic detrapping constant $\rho$ is readily calculated. Then, the kinetic trapping constant $k$ is evaluated from the equilibrium constant $K=k / p=\exp \left(\Delta G^{\prime} / R T\right)$, where $\Delta G^{\prime}$ is the uncorrected value of the free energy of trapping, directly given by the software used in this work. The hydrogen concentration beneath the input surface is calculated from the steady state permeation flux as $c_{0}=i_{\mathrm{H}, s} L /(D F)$. With these data the parameters $\lambda$ and $\rho$ are obtained. The different sets of parameters $(\lambda, \rho, \mu)$ corresponding to the different test temperatures of the API 5L X60 steel, which are presented in Table 5, are used as input data for the software of [7] to calculate theoretical permeation curves. We have observed that the critical value of $\mu$ to achieve local equilibrium conditions ranges from 100 to 1000 depending on the value of the other parameters, especially on the value of $\rho$. Therefore, a value of $\mu=1000$ is chosen to ensure the local equilibrium condition. The permeation transients calculated

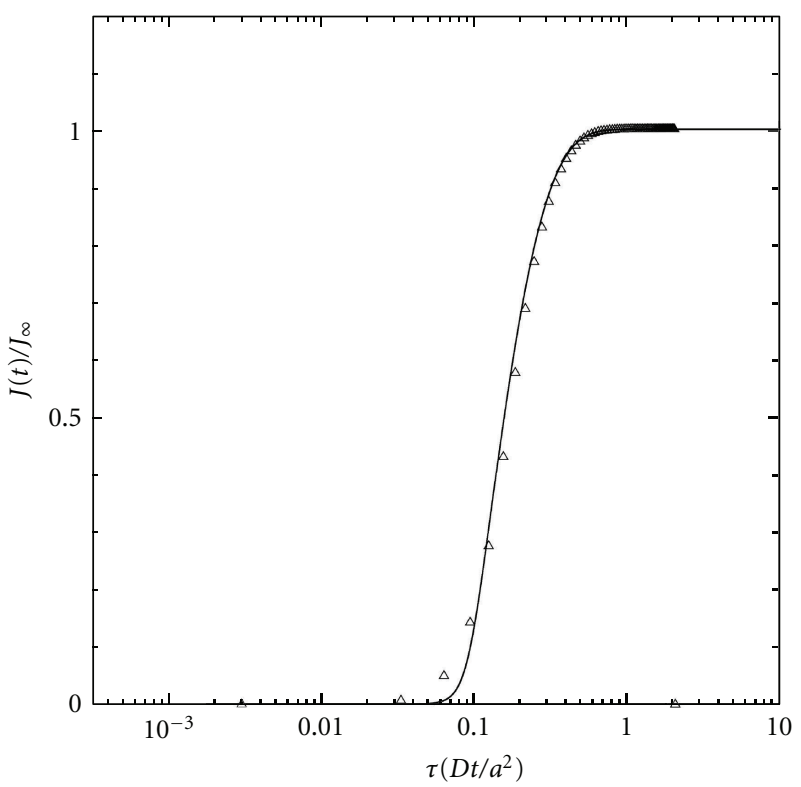

FIGURE 11: Comparison between the theoretical transients of the API 5L X60 steel in the AR condition at $50^{\circ} \mathrm{C}$, calculated with the present software (empty triangles) and with the software of [7] (filled line). $J_{\infty}$ is the hydrogen flux in the steady state, $D=D_{\text {app }}$ and $a=0.157 \mathrm{~cm}$.

in this way with the software of [7] are presented in Figures 10,11 , and 12 together with the theoretical permeation transients calculated with the software (restricted to local equilibrium) used in the present work. The agreement between both calculated transients is quite good, especially when the $\rho$ value is around 3. When $\rho$ increases to 16 or 18 , a small but unimportant discrepancy is observed. This discrepancy is attributed to the inaccuracy of the numerical method itself, in each case. This procedure is employed as a validation of the present software by contrast with other calculation method developed independently.

\section{Conclusions}

A method to calculate permeation transients and fit them to experimental data has been developed. The method assumes the presence of saturable traps in local equilibrium with lattice hydrogen. Numerical approaches dealing with hydrogen permeation in steels have been the subject of many studies. However, the present approach introduces the following innovating features

(i) Weak traps are taken into account via the apparent diffusion coefficient. This improves the software performance since the adoption of an apparent diffusion coefficient lower than the lattice diffusion coefficient allows increasing the length of the time steps of the numerical method. We notice that this procedure implies a change in the reference state for hydrogen, and this leads to a correction in the calculated free energy of trapping. 
TABLE 5: Diffusion parameters for the API 5L X60 AR steel condition from calculated rising transients.

\begin{tabular}{|c|c|c|c|c|c|c|c|}
\hline \multicolumn{8}{|c|}{ Rising transient } \\
\hline Temperature & $D_{\text {app }}$ & $i_{\mathrm{ss}}$ & & Reference [7] & & $\Delta G^{\prime}$ & $N$ \\
\hline${ }^{\circ} \mathrm{C}$ & $\mathrm{cm}^{2} / \mathrm{s}$ & $\mathrm{A} / \mathrm{cm}^{2}$ & $\lambda$ & $\rho$ & $\mu$ & $\mathrm{KJ} / \mathrm{mol}$ & $\mathrm{mol} \mathrm{H} / \mathrm{cm}^{3}$ \\
\hline 30 & $4.27 \times 10^{-6}$ & $1.28 \times 10^{-7}$ & 3138 & 3.36 & 1000 & -48.4 & $1.45 \times 10^{-8}$ \\
\hline 50 & $7.53 \times 10^{-6}$ & $3.13 \times 10^{-7}$ & 2074 & 15.93 & 1000 & -53.8 & $4.24 \times 10^{-9}$ \\
\hline 70 & $1.73 \times 10^{-5}$ & $6.12 \times 10^{-7}$ & 1393 & 18.74 & 1000 & -56.9 & $3.08 \times 10^{-9}$ \\
\hline
\end{tabular}

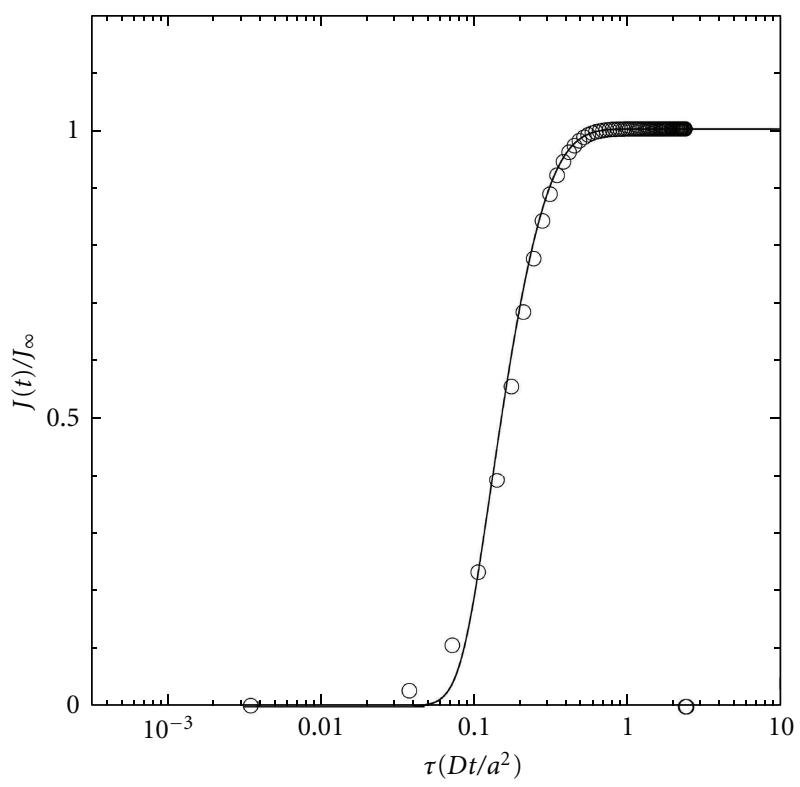

Figure 12: Comparison between the theoretical transients of the API 5L X60 steel in the AR condition at $70^{\circ} \mathrm{C}$, calculated with the present software (empty circles) and with the software of [7] (filled line). $J_{\infty}$ is the hydrogen flux in the steady state, $D=D_{\text {app }}$ and $a=$ $0.157 \mathrm{~cm}$.

(ii) The fitting procedure is made automatic by using the least squares fitting method and allowing selected parameters to vary.

(iii) The software is able to consider the presence of more than one type of trapping site in the material, in addition to the weak traps [15].

(iv) The present method has been validated by comparison with the method of Ramunni et al. [7], where the parameters for the latter are chosen in order to reach the local equilibrium condition.

(v) The most remarkable fact of the present work is that the trapping parameters are calculated univocally. With the model in [7] the fit is performed by varying two parameters, namely, $\rho$ and $\lambda / \mu$. The best fit is achieved for a one-dimension family in the two-dimensional space of these parameters. Then, the fit alone dose not univocally determine the experimental parameters. Meanwhile the Bruzzoniś model fixes the $\rho$ parameter. Once $\rho$ is fixed the fit fixes $\lambda / \mu$ hence the relevant trapping parameters are univocally determined.
Here we simulate permeation transients in order to establish a range of free energies of trapping that can be assessed through a single permeation transient. The results show that typical hydrogen permeation experiments (gas phase charging with a hydrogen partial pressure of $1 \mathrm{bar}$ and test temperatures between $30^{\circ} \mathrm{C}$ and $70^{\circ} \mathrm{C}$ ) are able to determine free energies of trapping in the approximate range of $-35 \mathrm{~kJ} / \mathrm{mol}$ to $-70 \mathrm{~kJ} / \mathrm{mol}$. Since local equilibrium is assumed, the three trapping parameters of McNabb and Foster are reduced to two. Then, when only one type of traps is considered, the model uses just three adjustable parameters to simulate (or fit) the permeation transient. The present method with one type of high energy traps describes very well the main characteristics of hydrogen trapping revealed by permeation tests on an API 5L X60 steel in the AR condition. Although the method has been applied here to a particular steel, it is applicable to iron base alloys in general. We remark that, since local equilibrium is assumed, the three trapping parameters of McNabb and Foster $(\lambda, \rho, \mu)$ are reduced to two: the trap density of strong traps $N$ and their free energy of trapping $\Delta G$. Then, when only one type of traps is considered, the present method uses just three adjustable parameters $(D, N, \Delta G)$ to calculate the permeation transient.

\section{Acknowledgments}

The authors gratefully acknowledge financial support from El Consejo Nacional de Investigaciones Cientficas y Tecnolgicas CONICET, Project no. PIP 00965/10 and from La Agencia de Promocin Cientfica y Tecnolgica, Project PAE 22590. The authors also acknowledge discussions with A. M. F. Rivas.

\section{References}

[1] A. McNabb and P. K. Foster, "A new analysis of the diffusion of hydrogen in iron and ferritic steels," Transactions of the Metallurgical Society of AIME, vol. 227, pp. 618-626, 1963.

[2] G. R. Caskey and W. L. Pillinger, "Effect of trapping on hydrogen permeation," Metallurgical and Materials Transactions A, vol. 6, no. 2, pp. 467-476, 1975.

[3] P. G. Thomas and E. J. Stern, "Efficient numerical modelling of hydrogen diffusion with trapping," Journal of Materials Science, vol. 16, no. 11, pp. 3122-3130, 1981.

[4] H. H. Johnson and R. W. Lin, "Hydrogen and deuterium trapping in iron," in Hydrogen Effects in Metals, Metallurgical Society of AIME, I. M. Bernstein and A. W. Thompson, Eds., pp. 3-25, 1981. 
[5] R. A. Oriani, "The diffusion and trapping of hydrogen in steel," Acta Metallurgica, vol. 18, no. 1, pp. 147-157, 1970.

[6] D. H. Ferris and A. Turnbull, "Analysis of reversible and irreversible hydrogen trapping in metals," NPL Report DMA (A) 154, 1988.

[7] V. P. Ramunni, T. D. P. Coelho, and P. E. V. de Miranda, "Interaction of hydrogen with the microstructure of lowcarbon steel," Materials Science and Engineering A, vol. 435436, pp. 504-514, 2006.

[8] F. G. Wei, T. Hara, and K. Tsuzaki, "Nano-precipitates design with hydrogen trapping character in high strength steels," in Effects of Hydrogen on Materials, Proceedings of the 2008 International HydrogenConference, B. Somerday, P. Sofronis, and R. Jones, Eds., pp. 448-455, ASM International, 2009.

[9] E. Riecke and K. Bohnenkamp, "Über den Einfluß von Gitterstörstellen in Eisen auf die Wasserstoffdiffusion," Zeitschrift für Metallkunde, vol. 75, pp. 76-81, 1984.

[10] R. S. Chaudhary and E. Riecke, "Untersuchungen zum Einfluß des Stahlgefüges auf die station re Wasserstoffpermeation," Werkstoffe und Korrosion, vol. 32, no. 2, pp. 66-72, 1981.

[11] P. Castaño Rivera and P. Bruzzoni, "Interacción hidrgeno metal en un acero para gasoductos," in Memorias Tercer Congreso Nacional y Segundo Congreso Iberoamericano de Hidrgeno y Fuentes Sustentables de Energía, 2009.

[12] A. J. Kumnick and H. H. Johnson, "Steady state hydrogen transport through zone refined irons," Metallurgical and Materials Transactions A, vol. 6, no. 5, pp. 1087-1091, 1975.

[13] M. A. V. Devanathan and Z. Stachurski, "The absorption and diffusion of electrolytic hydrogen in palladium," Proceedings of the Royal Society A, vol. 270, no. 1340, pp. 90-102, 1962.

[14] E. Riecke, "Untersuchungen zum Eifluß des Stahlgefüges auf die stationäre Wasserstofpermeation," Werkstoffe und Korrosion, vol. 32, pp. 66-72, 1981.

[15] P. Castaño Rivera, V.P. Ramunni, and P. Bruzzoni, "Hydrogen trapping in an API 5L X60 steel," Corrosion Science, vol. 54, pp. 106-118, 2012. 

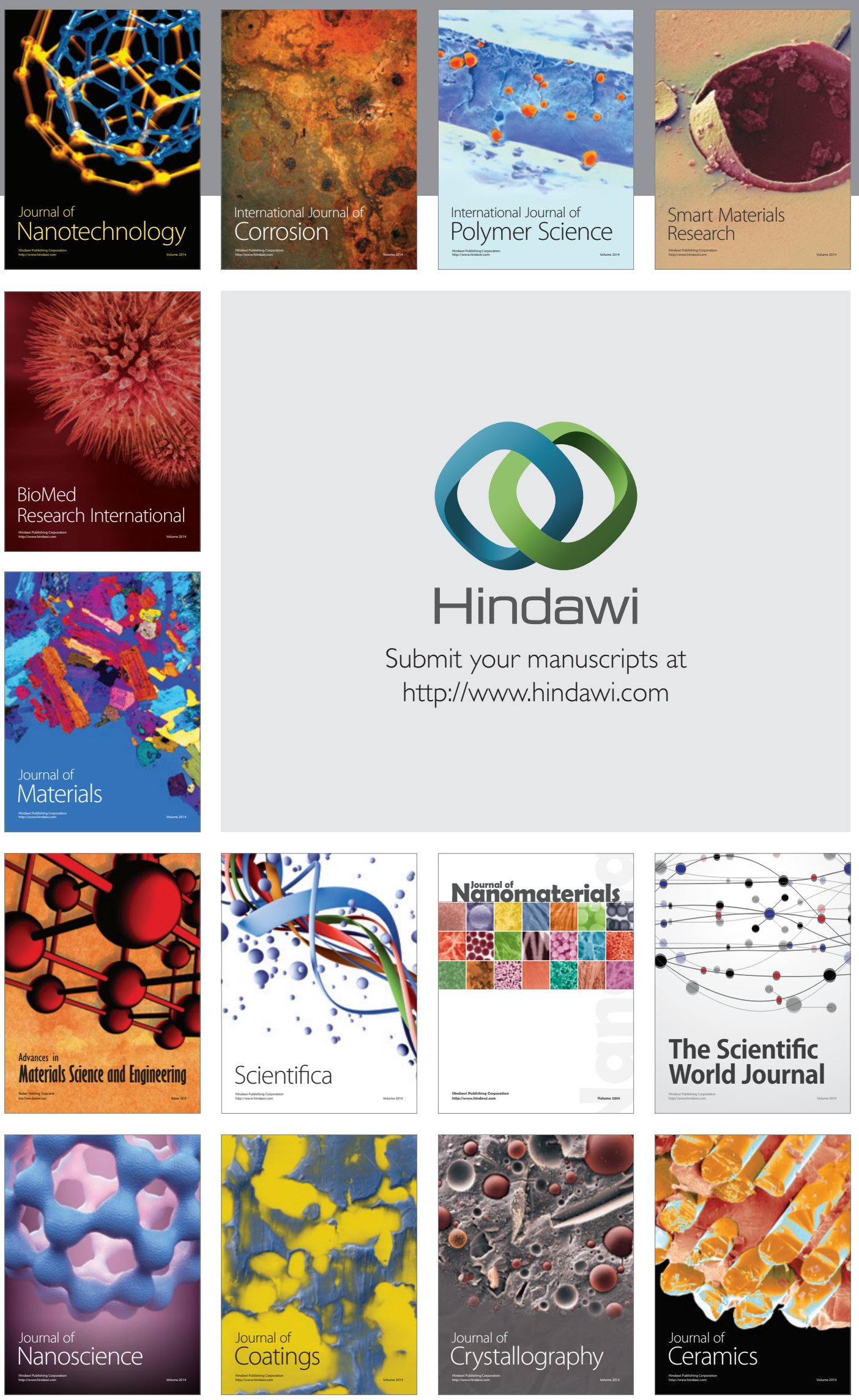

The Scientific World Journal

Submit your manuscripts at

http://www.hindawi.com

\section{World Journal}

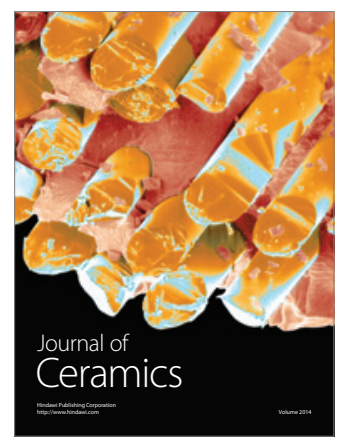

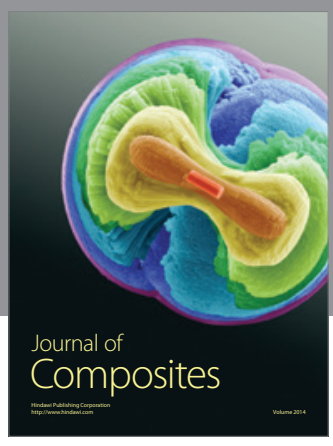
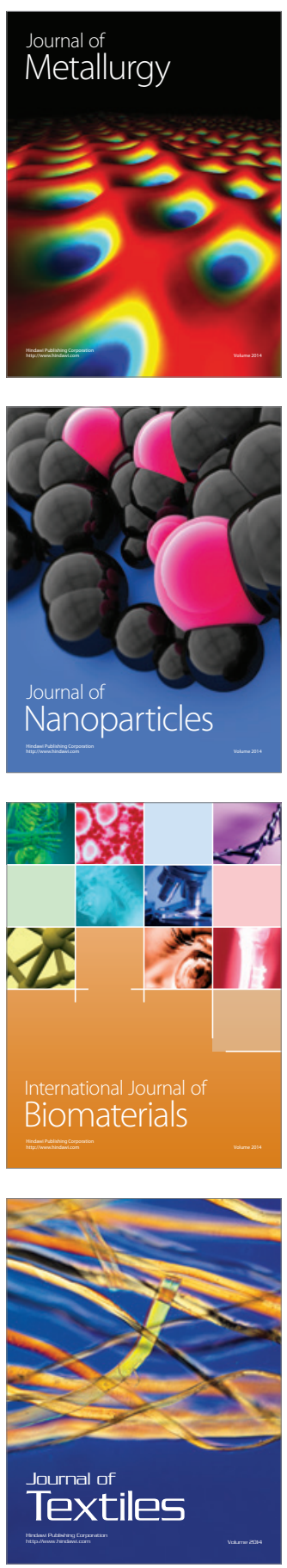